\title{
Hábitos de ingesta hídrica e miccionais e sua associação com infecção do trato urinário em jovens de uma escola da rede pública do Distrito Federal
}

\author{
Mictional and water intake habits and its association with urinary tract infection in young \\ people of a public school in Federal District
}

Hábitos de ingesta hídrica y miccionales y su asociación com infección del tracto urinário em jóvenes de uma escuela de enseñanza pública del Distrito Federal

Silvio Rosa de Freitas Júnior ${ }^{*}$, Renan Lucas Moura Lima da Silva ${ }^{1}$, Érico Augusto Rosas de Vasconcelos'.

\section{RESUMO}

Objetivo: Descrever o perfil dos jovens de uma escola da rede pública do Distrito Federal (DF) em relação ao conhecimento acerca de infecções do trato urinário e o comportamento de micção e de ingesta hídrica dos mesmos. Método: Estudo transversal, quantitativo e descritivo. A coleta de dados compreendeu um questionário semiestruturado autoaplicável contendo 9 questões. O estudo foi realizado em uma escola da rede pública do DF com jovens na faixa etária de 14 a 18 anos. Foram excluídos aqueles que sabidamente referiram alguma disfunção de origem neurológica ou urológica que afetasse o sistema urinário. Resultados e discussão: $O$ presente estudo mostrou que $70,7 \%$ da amostragem tem conhecimento do que é infecção do trato urinário e que $51,2 \%$ sabe quais são as principais causas deste tipo de infecção, mesmo que, em contrapartida, estes apresentem comportamentos de risco para ocorrência de infecções do trato urinário (ITU's). Conclusão: Foi possível identificar no estudo comportamentos de risco dos jovens no que diz respeito à ingestão hídrica e à micção, frente às infecções do trato urinário.

Palavras-chave: Hábitos, Ingestão de líquidos, Micção.

\section{ABSTRACT}

Objective: This work aimed to describe the profile of the students of a public school in Distrito Federal (DF) in relation to knowledge about urinary tract infections, mictional and water intake behavior. Methodology: Crosssectional, quantitative and descriptive study. A self-administered semistructured survey containing 9 questions was applied to the data acquisition. The study was accomplished at a public school in DF with students aged from 14 to 18 years. Those whom know to have reported some dysfunction of neurological or urological origin that affected the urinary system were excluded from the study. Results and Discussion: Here we show that $70.7 \%$ of the students encompassing the sample are aware of what is an urinary tract infection, and $51,2 \%$ of them knows what are the main causes of this type of infection. In contrast, they still have the occurrence of urinary tract infections (UTIs). Conclusion: It was possible to identify a risky behavior among students in relation to water intake and mictional habits, which could lead to urinary tract infections.

Key words: Habits, Drinking, Urination.

\section{RESUMEN}

Objetivo: Describir el perfil de los jóvenes de una escuela de enseñanza pública del Distrito Federal (DF) en relación con el conocimiento de las infecciones del tracto urinario y el comportamiento de micción y de ingesta hídrica de los mismos. Método: Estudio transversal, cuantitativo y descriptivo. La recolección de datos comprendió un cuestionario semiestructurado auto aplicable que contenía 9 cuestiones. El estudio fue

${ }^{1}$ Centro Universitário Euro-Americano (UNIEURO), Brasília (DF). *E-mail: juniorfreitas201248@hotmail.com 
realizado en una escuela pública del DF con jóvenes en el grupo de edad de 14 a 18 años. Eliminados aquellos que sabidamente refirieron alguna disfunción de origen neurológico o urológico que afecte el sistema urinario. Resultados y Discusión: El presente estudio posibilitó identificar en la muestra que el 70,7\% tiene conocimiento de lo que es infección del tracto urinario y que el $51,2 \%$ sabe cuáles son las principales causas de este tipo de infección aunque en contrapartida éstos todavía posean comportamientos de riesgos la ocurrencia de infecciones del tracto urinario (ITU's). Conclusión: Fue posible identificar en el estudio, comportamientos de riesgos de los jóvenes frente a las infecciones del tracto urinario.

Palabras clave: Hábitos, Ingestión de líquidos, Micción.

\section{INTRODUÇÃO}

A infecção sintomática do trato urinário (ITU) é a segunda infecção mais comum na população, atrás somente das infecções respiratórias. Essa patologia é definida, por grande parte dos autores, como a colonização microbiana com invasão tecidual de qualquer parte do trato urinário, desde a uretra até os rins. A invasão dos microrganismos pode alcançar o trato urinário por meio de três vias: ascendente pela uretra, via hematogênica e linfática (BRAOIOS A, et al., 2009).

De acordo com estudo de Oliveira SM e Santos LLG (2018), no que se refere a infecção do trato urinário, inicialmente a pessoa apresenta inflamação na uretra que, na ausência de tratamento adequado acomete a bexiga (cistite) e os ureteres (uretrite). Possivelmente, os agentes infecciosos irão deslocar-se até os rins que de maneira severa, poderá ser a causa de morte por infecção generalizada (septicemia). Vemos ainda, que este quadro infeccioso, acomete pessoas de ambos os sexos e de todas as idades porém, mulheres jovens compõem o principal grupo de risco. Este estudo, realizado em Portugal, nos mostra que, através da coleta de 385 uroculturas positivas, 334 eram de indivíduos do sexo feminino e 53 do sexo masculino. Observamos ainda, que as ITU's são responsáveis por grande parte das consultas clínicas e tem gerado elevados custos à economia global (OLIVEIRA SM e SANTOS LLG, 2018).

As infecções do trato urinário são classificadas como complicadas ou não complicadas. As complicadas apresentam maior risco de falha terapêutica e ocorre no aparelho urinário com alterações estruturais ou funcionais como, por exemplo, a Pielonefrite (infecção alta), pois, em geral, resulta da ascensão de microrganismos do trato urinário inferior e está fortemente associada a cálculos renais e à presença de sintoma, como a dor. Por outro lado, as não complicadas apresentam características mais brandas e podem ser assintomáticas, como a Cistite, que é uma infecção baixa. A infecção alta acomete os rins enquanto a baixa se estabelece prioritariamente na bexiga (LOPES HV e TAVARES W, 2005).

As bactérias mais comuns relacionadas a ITU são da família Enterobacteriaceae, sendo a espécie Escherichia coli a mais comum e responsável desde quadros clínicos não complicados até aos mais complicados e crônicos (OLIVEIRA SM e SANTOS LLG, 2018).

De acordo com Gupta, et al. (2001), o diagnóstico realizado por meio de urocultura com o antibiograma é considerado o método mais eficaz para detecção do agente causador e propicia um tratamento adequado focado no agente etiológico causador da infecção. Além disso, o início do tratamento imediato com antimicrobianos é importante na prevenção de cicatriz renal de longo prazo. Para Simões AC e Oliveira EA (2015), o primeiro passo para o diagnóstico da ITU é o histórico de saúde, que inclui perguntas sobre infecções primárias ou recorrentes, consumo de bebidas e hábitos de micção. Geralmente pacientes com alguma disfunção miccional apresentam baixa frequência urinária (TRAPP C, et al., 2013).

Para Oliveira SM e Santos LLG (2018), as ITU's estão mais prevalentes na população do sexo feminino, como evidenciado em um estudo realizado em Mato Grosso, onde se observou que, de um o total de 598 exames de uroculturas realizados por laboratório, 118 (19,73\%) foram positivos para ITU. Ademais, destes 118 pacientes $93(78,81 \%)$ eram do sexo feminino. Em estudo de XU D, et al. (2016), realizado com enfermeiras chinesas, $55 \%$ delas não se consideravam em risco por seus hábitos miccionais e, em 
contrapartida, $67.5 \%$ referiam apresentar pelo menos um sintoma de infecção no trato urinário inferior (STUI) durante a pesquisa. Os dados apresentados neste estudo, nos leva a crer que, por vezes, o indivíduo não acredita que seus hábitos miccionais são de risco e, por conseguinte, aumentam a probabilidade de ocorrência de complicações, como o desenvolvimento de STUI ou, até mesmo, infecções do trato urinário (ITU) (XU D, et al. 2016).

Atualmente, a Sociedade Brasileira de Alimentação e Nutrição (2016) estabelece um valor padrão para a ingesta hídrica diária e o valor preconizado para os jovens na faixa etária de 14 a 18 anos é de 2,6Litros/dia para meninos e 1,8Litros/dia para meninas. Afirma-se, ainda, que o bom hábito de micção favorece a eliminação de microrganismos pela urina, evitando-se, assim, infecções. Em estudo de Das RN, et al. (2012), realizado com jovens estudantes do quinto ano de uma Universidade do sul da Austrália, foi observado dado positivo quanto à ingesta de líquido. Em sua amostra, a média foi de $2.600 \mathrm{ml}$ mesmo não sendo período de verão no país durante a investigação. $O$ alto valor observado pode ter se dado pelo fato de que a amostra, em sua grande parte, acreditava que a quantidade ideal de água diária deveria ser de pelo menos oito copos em mls. Porém, a generalização dos dados desse estudo para a realidade brasileira torna-se inviável, uma vez que a ingesta de líquidos é também um hábito, ou seja, sofre influências culturais e até mesmo climáticas. Desta forma, Rossi L, et al. (2010) destaca que o Brasil tem predominância de clima tropical e temperaturas médias elevadas durante a maior parte do ano, o que requer maior hidratação por parte dos brasileiros. Portanto, uma avaliação própria para os brasileiros que residem em regiões de baixa umidade, como o caso do Distrito Federal, faz-se necessária, considerando também o fato de que são totalmente deficientes os estudos brasileiros voltados a essa temática. Tendo isso em vista, faz-se imprescindível conhecer os hábitos miccionais e de ingesta hídrica dos brasileiros de regiões como o Distrito Federal, bem como se há ocorrências de complicações oriundas de comportamentos de risco como as infecções do trato urinário.

Dado o exposto, cabe ressaltar a importância de um estudo aprofundado direcionado à investigação de hábitos miccionais e de ingesta hídrica dos jovens, que são a parcela da população mais susceptível e menos informada na prevenção de ITU's. Para Trapp C, et al. (2013), as disfunções miccionais infantis podem acometer crianças em qualquer idade, porém, ficam mais evidentes após a desfralda até a juventude. Em suma, o presente estudo objetiva conhecer o perfil de Jovens de uma escola da rede pública do Distrito Federal em relação ao conhecimento acerca de infecções do trato urinário, bem como traçar os comportamentos de micção e ingesta hídrica.

\section{MÉTODOS}

\section{Tipo de estudo}

Trata-se de um estudo transversal com abordagem quantitativa e descritiva.

\section{Coleta de dados}

Para o desenvolvimento da pesquisa, foi utilizado questionário autoaplicável desenvolvido com perguntas objetivas a respeito dos hábitos miccionais, de ingesta hídrica e conhecimento prévio de infecções do trato urinário (ITU).

O instrumento foi composto por quatro eixos: o primeiro diz respeito à identificação, no qual foram colhidos os dados de caracterização do indivíduo. O segundo incluiu o conhecimento dos jovens a respeito de infecção do trato urinário, de ocorrência de ITU no último ano e influência climática sob a ingesta. $O$ terceiro eixo foi composto por informações sobre ingesta hídrica, incluindo o volume médio de consumo de água e de outras bebidas durante o dia. $O$ quarto eixo contemplou questões relativas aos hábitos miccionais, tais como frequência miccional diária (sendo estes descritos como 1-3, 4-7, 8-10 ou < 10 vezes).

A coleta de dados ocorreu nos meses de março e abril de 2019 no Centro de Ensino Médio 04 de Ceilândia, uma escola de ensino médio da rede pública do DF. Para prévia validação do instrumento de coleta de dados, foi aplicado teste piloto com uma pequena parcela da amostra a fim de confirmar se as questões estavam de fato compreensíveis e claras. Participaram da pesquisa os alunos do segundo e do 
terceiro ano do ensino médio com idades entre 14 e 18 anos, devidamente matriculados na escola e que assinaram previamente o Termo de Consentimento Livre e Esclarecido. A pesquisa foi realizada com os alunos do turno matutino e nos horários disponibilizados pela direção da instituição, com o fito de que a pesquisa não interrompesse as atividades escolares. Foram excluídos aqueles que sabidamente referiram alguma disfunção de origem urológica ou neurológica que afetasse o sistema urinário. No total, foram 588 alunos distribuídos em 14 salas durante o turno da manhã sendo que a pesquisa foi apresentada a todos e apenas 41 tiveram interesse em participar, estando estes também inclusos no perfil exigido para amostra.

\section{Análise de dados}

A análise dos dados foi realizada utilizando-se o aplicativo Microsoft office Excel 2013.

\section{Aspectos éticos}

O projeto de pesquisa referente ao presente estudo foi previamente submetido ao Comitê de Ética e Pesquisa do Centro Universitário Euro-Americano (CEP/UNIEURO) e aprovado com o número de parecer 3.193.801. Em todas as etapas da pesquisa, foram considerados os preceitos estabelecidos na resolução $466 / 2012$, que delibera sobre pesquisa com seres humanos. Os pesquisadores mantiveram o respeito aos sujeitos da pesquisa em sua total dignidade e autonomia, assegurando sua vontade de contribuir e permanecer ou não na pesquisa, por meio de manifestação expressa, livre e esclarecida. Dessa forma, participaram da pesquisa apenas os alunos que assinaram, juntamente com seus responsáveis, os Termos de Consentimento Livre e Esclarecido.

\section{RESULTADOS E DISCUSSÃO}

A amostra foi composta por ambos os sexos, sendo 29 meninas $(70,7 \%)$ e 12 meninos $(29,2 \%)$. Do total de 29 meninas participantes, 4 apresentaram infecções urinárias nos 12 últimos meses, sendo que uma teve apenas uma ocorrência e três tiveram duas ocorrências no período (Figura 1).

Figura 1 - Número de ocorrência de ITU em período de 12 meses nas meninas. Observa-se o percentual de ocorrências de infecção do trato urinário em 0,1 ou 2 vezes nas meninas do estudo.

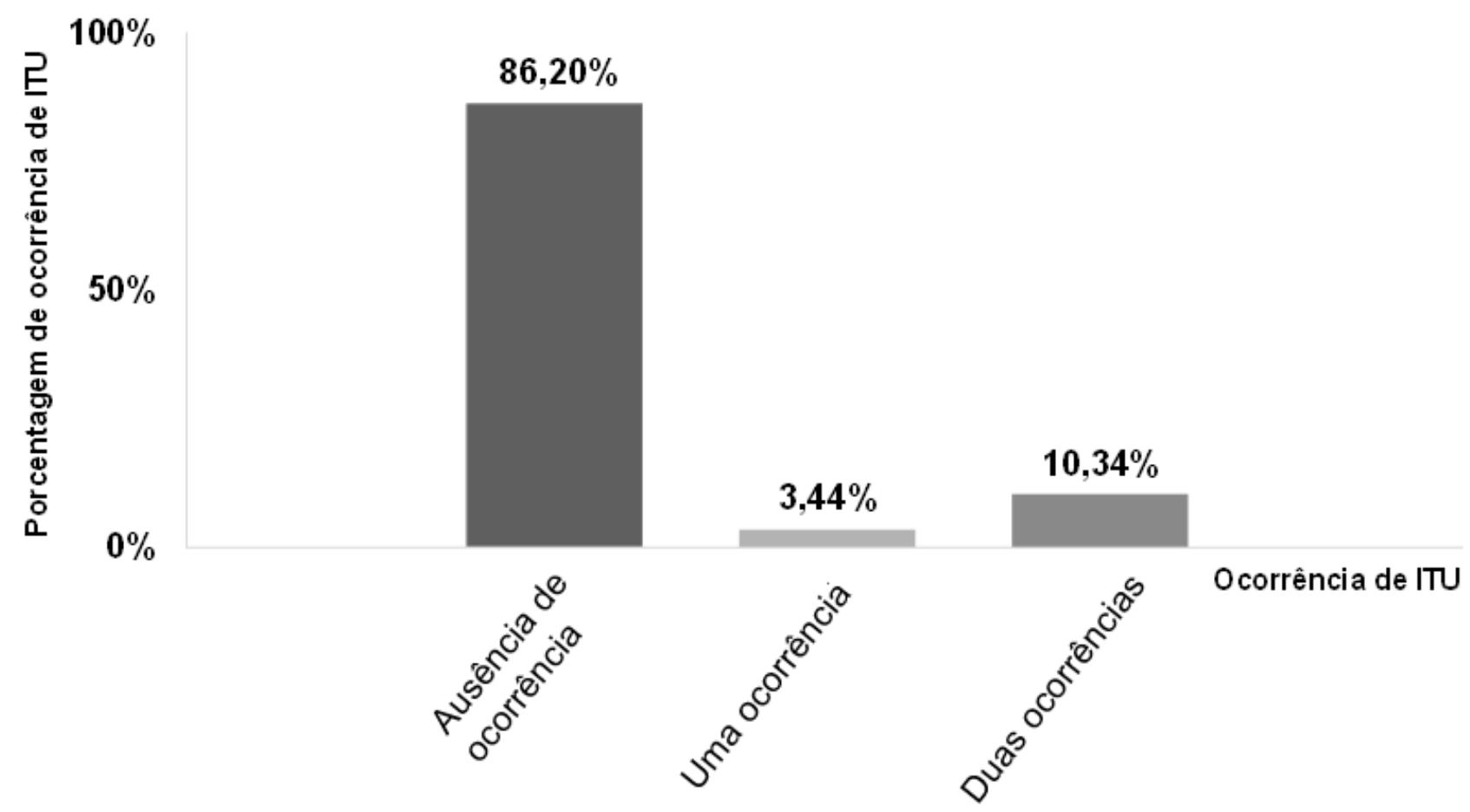

Fonte: Dados da pesquisa, 2019. 
Para Gupta, et al. (2001), a alta incidência das infecções urinárias, seu caráter brando e a necessidade de iniciar o tratamento antes da finalização dos exames microbiológicos implicam a frequente adoção de tratamento empírico. Os dados mostram que a ocorrência de ITU é prevalente no sexo feminino, estando ainda em concordância com outros estudos, como de Oliveira SM e Santos LLG (2018), no qual se prova que o grupo feminino é a população que apresenta maior susceptibilidade a este tipo de ocorrência devido a fatores como higienização errônea da genitália, cateterismo urinário, estado gravídico e, até mesmo, condições anatômicas como a uretra mais curta e sua maior proximidade da vagina com o ânus. Existem também outros fatores que tornam as mulheres mais propensas à ocorrência de ITU: os episódios prévios de cistite, o ato sexual, o uso de certas geleias espermicidas e a quantidade de gestações (SOUZA AESD, 2009).

O estudo mostra que, nos meninos, nenhuma ITU foi diagnosticada e que $70,7 \%$ dos entrevistados afirmam ter conhecimento do que é infecção do trato urinário e $51,2 \%$ sabem quais os fatores de risco que favorecem o surgimento de ITU (Tabela 1). Em contrapartida, vemos que alguns comportamentos ainda os tornam propensos à ocorrência de ITU, tais como a ingesta hídrica diária insuficiente a necessidade corporal e a associação da ingesta com mudanças climáticas.

Tabela 1 - Caracterização dos fatores de risco para ocorrência de ITU em jovens do Ensino Médio.

\begin{tabular}{lcc}
\hline 1. CONHECIMENTO ACERCA DE INFECÇÃO DO TRATO URINÁRIO & SIM & NÃO \\
\hline \hline 1.1. Sabe o que é infecção do trato urinário? & $70.70 \%$ & $29.20 \%$ \\
1.2. Sabe quais são as principais causas de infecção do trato urinário? & $51.20 \%$ & $48.70 \%$
\end{tabular}

\section{COMPORTAMENTO DE INGESTA HÍDRICA}

$\begin{aligned} & \text { 2.1. Acredita que a ingesta de líquidos está relacionada a temperatura } \\ & \text { climática? }\end{aligned} \quad 68.20 \% \quad 31.70 \%$
2.2. Acredita que deve-se obrigatoriamente ingerir uma quantidade específica de líquidos diariamente?

$97.50 \% \quad 2.40 \%$

\section{COMPORTAMENTO MICCIONAL}

\begin{tabular}{l}
\hline 3.1. Quando está com a bexiga cheia, costuma segurar a vontade de urinar? \\
$\begin{array}{l}\text { 3.2. Você já deixou de ingerir líquidos para passar a ir menos vezes ao } \\
\text { banheiro? }\end{array}$ \\
\hline
\end{tabular}

Fonte: Dados da pesquisa, 2019.

Tendo em vista que a quantidade de água necessária para o bom funcionamento do organismo possa variar devido a diversos fatores, devemos considerar, segundo a Sociedade Brasileira de Alimentação e Nutrição (2016) e o Instituto de Medicina dos Estados Unidos (IOM), que, para jovens do sexo masculino e feminino com idades entre 14 e 18 anos, o valor diário recomendado é 2,6 Litros/dia ou 11 copos e 1,8 Litros/dia ou 8 copos respectivamente (AZEVEDO PS, et al., 2016). Além disso, 68,2\% afirmam que a ingesta está relacionada a alterações climáticas (Tabela 1). 
De acordo com Rossi L, et al. (2010), o Brasil é uma país localizado em região tropical e caracterizado por apresentar grandes variações climáticas devido a suas proporções continentais, com associação de altas temperaturas na maior parte do ano. Sendo assim, considera-se imprescindível a abordagem dessa população com questões de educação em saúde para que a ITU seja prevenida a partir da eliminação dos fatores de risco inerentes a sua ocorrência. Para Lorenzo A e Liaño $F$ (2017), as mudanças climáticas afetam expressivamente a saúde humana, por consequência, as ondas de calor, acompanhadas de aumento da transpiração e perda de fluídos pelo organismo, em associação com situações de sensação alterada da sede e/ou diminuição da ingesta de água, aumentam a morbidade e mortalidade, bem como o risco de insuficiência renal e hospitalização devido a doenças renais. Azevedo PS, et al. (2016) considera que, a ingestão de água é fundamental para se suprir as necessidades diárias do organismo, pois, ao decorrer do dia, todos nós apresentamos perdas de água insensíveis: em torno de $450 \mathrm{~mL}$ em média; enquanto que as perdas por meio do suor são extremamente variáveis e dependentes de fatores metabólicos e ambientais. A perda de água pelas fezes, em geral, mantém-se constante, em torno de $100 \mathrm{~mL}$ a $200 \mathrm{~mL}$ por dia, excetuando casos de diarreia. Deve-se considerar que a urina é o principal meio pelo qual ocorre perda de água, e a quantidade produzida é bastante variável, dependendo de diversos fatores, como, por exemplo, a quantidade de água ingerida no dia ou perdida através dos outros meios, como o suor. Em geral, a produção diária de urina permanece em torno de $1.000 \mathrm{~mL}$ a $2.000 \mathrm{~mL}$. Desse modo, é vital consumir água em uma quantidade adequada para manter a homeostase, pois, em concordância com resultados deste artigo, as crianças consomem menos líquidos do que o recomendado, persistindo nelas um estado de sub-hidratação que pode afetar o nível de atenção, o desempenho escolar, o humor e a capacidade cognitiva (ARREDONDO JL, et al., 2017).

Pode-se observar que a baixa ocorrência de ITU na amostra se dá pelo fato de que a população alega ter um bom comportamento de ingesta hídrica, pois $97,5 \%$ afirmou que se deve, obrigatoriamente, ingerir uma quantidade específica de líquidos diariamente e apenas $2,4 \%$ afirma desconhecimento sobre isto (Tabela 1). Porém, tais dados se contradizem no questionamento posterior, em que é perguntado a quantidade de líquido ingerida diariamente. Nesse sentido, 68,2\% afirmam ingerir quantidade inferior a 1,2 Litro/dia (Tabela 2), sendo que se para Azevedo PS, et al. (2016), preconiza-se o valor diário recomendado para meninos de 2,6Litros/dia ou 11 copos, e para meninas 1,8 Litros/dia ou 8 copos.

Tabela 2. Frequência de ingesta de líquidos diária.

\section{Quanto de líquido (água e derivados) você ingere por dia? (Considere 1 copo equivalente a $200 \mathrm{~m} / / \mathrm{dia}$ ).}

não bebe $(4,89 \%) \quad 1$ a $3 \operatorname{copos}(26,9 \%) \quad 4$ a $6 \operatorname{copos}(36,5 \%) \quad$ mais que $6 \operatorname{copos}(31,7 \%)$

Fonte: Dados da pesquisa, 2019.

Conclui-se, então, que os jovens possuem conhecimento incorreto a respeito da quantidade ideal a ser ingerida diariamente, ou que sabem, porém não possuem o hábito correto. Tal fato reforça mais ainda o papel dos profissionais de saúde em questões relacionadas à educação e ao incentivo de adotar-se hábitos saudáveis. Cabe a estes profissionais fazer com que a população reconheça os sintomas provocados pela falta de água no organismo, como sede, fadiga, tontura, indisposição e desatenção, que ficam mais evidentes conforme a intensificação da falta de água. Além disso, a ITU pode ser assintomática como no caso da cistite que ocorre na bexiga, e sintomática, quando o indivíduo apresentar a pielonefrite que acomete os rins, e dentre os sintomas terá: dor lombar, náuseas e vômitos, aumento da frequência e urgência em urinar (SOUZA AESD, 2009).

O European Journal of Nutrition mostra a transcendência destas características de deficiente ingesta hídrica vista na população brasileira em comparação a estudos realizados em 13 diferentes países. No México, em uma análise de crianças com faixa etária de 1 a 18 anos, verificou-se que $80 \%$ delas possuem 
ingesta hídrica inferior ao considerado adequado. No Brasil, na Argentina e no México, a contribuição de sucos e refrigerantes na ingesta hídrica diária é semelhante à de água, e, dentre os 13 países, o Brasil ocupou o segundo lugar em porcentagem de pessoas que consomem mais energia proveniente de bebidas açucaradas do que o recomendado. Portanto, os profissionais de saúde devem garantir um bom estado de hidratação na população infantil, bem como intervenções para reduzir o consumo de bebidas açucaradas durante a dia (AZEVEDO PS, et al., 2016).

Este estudo mostra que $48,7 \%$ dos jovens afirmam reter a vontade de urinar quando estão de bexiga cheia e $51,2 \%$ negam este comportamento. $34,1 \%$ afirmam deixar de ingerir líquidos para passar a ir menos vezes ao banheiro sendo que $17 \%$ dizem que quase sempre têm este comportamento e $29,2 \%$ somente às vezes (Tabela 1). Considerando toda a avaliação dos dados, nota-se que a maior parcela dos jovens, representada por $70,7 \%$, sabe o que é infecção do trato urinário e $51,2 \%$ afirma ter conhecimento dos fatores de risco para ITU. Além disso, possuem um bom comportamento de micção visto que $51,2 \%$ diz não reter vontade de urinar e $65,8 \%$ alega não deixar de ingerir líquidos para passar a ir menos vezes ao banheiro durante o dia. Entretanto, cabe destacar que ainda há comportamento propenso a risco de ITU na amostra, como relacionar a ingesta hídrica a alterações climáticas e a não ingesta hídrica necessária ao organismo diariamente.

\section{CONCLUSÃO}

Foi possível concluir através do presente estudo que o conhecimento acerca de infecções do trato urinário e fatores de risco para ITU pelos jovens é satisfatório. Porém, nota-se ainda a presença de comportamentos que colocam os jovens em estado de vulnerabilidade à ocorrência de ITU, como a insuficiente ingesta hídrica diária além da correlação desta ingesta com alterações climáticas. Observa-se ainda que a maior prevalência de ITU está sob a população do sexo feminino. Destaca-se ainda o reconhecimento e a importância da atuação do profissional de saúde na adoção de medidas de prevenção, diagnóstico e tratamento frente à susceptibilidade e à ocorrência dessa infecção através da educação continuada para com a população jovem e da criação de diferentes meios de intervenção frente às infecções do trato urinário. $O$ presente estudo conta com uma amostragem limitada, é recomendado que para estudos posteriores abordando esta mesma temática seja captado um quantitativo superior de amostra para que seja obtido resultados ainda mais precisos na comparação das vulnerabilidades com a ocorrência de infecções do trato urinário.

\section{REFERÊNCIAS}

1. ARREDONDO JL, et al. Água: la importancia de una ingesta adecuada en pediatría. Acta pediátrica de México, 2017; 38(2): 116124.

2. AZEVEDO PS, et al. Água, Hidratação e Saúde. Sociedade Brasileira de Alimentação e Nutrição (SBAN), 2016; 6-14.

3. BRAOIOS A, et al. Infecções do trato urinário em pacientes não hospitalizados: etiologia e padrão de resistência aos antimicrobianos. Jornal Brasileiro de Patologia e Medicina Laboratorial, 2009; 6: 449-456.

4. BRASIL. Ministério da Saúde, Secretaria de Atenção à Saúde, Departamento de Atenção Básica. Guia alimentar para a população brasileira. - $2^{\circ}$ ed, 1 reimpr - Brasília: Ministério da Saúde, 2014.

5. BRASIL. Resolução n 466, de 2012. Coleção de leis da República Federativa do Brasil, Brasília, DF, dezembro de 2012.

6. DAS RN, et al. Fluid intake and voiding; habits and health knowledge in a young, healthy population. Research and reports in urology, 2012; 4(9)

7. GUPTA, et al. Patient initiated treatment of uncomplicated recurrent tract infections in young women. Annals of internal medicine, 2001; 135(1): 9-16

8. LOPES HV, TAVARES W. Diagnóstico das infecções do trato urinário. Revista da Associação Médica Brasileira. São Paulo, 2005 51(6): 306-308.

9. LORENZO A, LIAÑO F. Altas temperaturas y nefrología: a propósito del cambio climático. Nefrología (Madrid), 2017; 37(5): 492500

10. OLIVEIRA SM, SANTOS LLG. Infecção do trato urinário: estudo epidemiológico em prontuários laboratoriais. Journal Health NPEPS, 2018; 3(1): 198-210.

11. ROSSI L, et al. Desidratação e recomendações para a reposição hídrica em crianças fisicamente ativas. Revista Paulista de Pediatria, 2010; 28(3): 337-345.

12. SIMÕES AC, OLIVEIRA EA. Update on the approach of urinary tract infection in childhood. Jornal de Pediatria (Versão em português), 2015; 91(6): S2-S10.

13. SOUZA AESD. Epidemiologia das infecções urinárias de pacientes atendidos em hospital público. Rev. para Med, 2009; 23(4)

14. TRAPP C, et al. Distúrbios da micção em crianças. Bol Cient Pediat, 2013; 2(2): 8-53.

15. XU D, et al. Toileting behaviour and related health beliefs among Chinese female nurses. International journal of clinical practice, 2016; 70(5): 416-423. 